\title{
Polynomial Datapath Optimization using Constraint Solving and Formal Modelling
}

\author{
Finn Haedicke ${ }^{\dagger}$, Bijan Alizadeh ${ }^{\ddagger}$, Görschwin Fey ${ }^{\dagger}$, Masahiro Fujita ${ }^{\ddagger}$, Rolf Drechsler ${ }^{\dagger}$ \\ ${ }^{\dagger}$ Institute of Computer Science, University of Bremen, 28359 Bremen, Germany \\ \{finn, fey, drechsle\}@informatik.uni.bremen.de \\ $\ddagger$ VLSI Design and Education Center (VDEC), University of Tokyo and CREST, Tokyo, Japan \\ alizadeh@cad.t.u-tokyo.ac.jp, fujita@ee.t.u-tokyo.ac.jp
}

\begin{abstract}
For a variety of signal processing applications polynomials are implemented in circuits. Recent work on polynomial datapath optimization achieved significant reductions of hardware cost as well as delay compared to previous approaches like Horner form or Common Sub-expression Elimination (CSE). This work 1) proposes a formal model for single- and multi-polynomial factorization and 2) handles optimization as a constraint solving problem using an explicit cost function. By this, optimal datapath implementations with respect to the cost function are determined. Compared to recent state-of-the-art heuristics an average reduction of area and critical path delay is achieved.
\end{abstract}

\section{INTRODUCTION}

Although polynomial expressions are frequently encountered in many applications such as computer graphics and Digital Signal Processing (DSP) domains, conventional high-level synthesis techniques are not able to manipulate polynomial expressions efficiently due to the lack of suitable optimization techniques for redundancy elimination over $\mathbb{Z}_{2^{n}}$. From a synthesis point of view, the designers often optimize such polynomial functions manually to achieve efficient Register-Transfer-Level (RTL) implementation. However this process can be both time consuming and error prone. As a result, developing high-level optimization and synthesis techniques is desirable to automate the design of custom datapaths from a behavioral description.

The design of computationally expensive embedded systems for multimedia and DSP applications starts with the algorithmic specification in a high level language such as MATLAB. This specification performs a sequence of arithmetic polynomial computations with integer variables of infinite bit-widths which is often implemented with fixed-point architectures. When refining algorithmic specifications to RTL descriptions, the RTL models are often implemented with fixed word-length datapath architectures. The polynomial computations are carried out over $n$-bit integers where the size of the entire datapath is kept constant by signal truncation. Hence modular polynomial optimization and synthesis should be supported. For example, consider $f_{1}=x+5 y, f_{2}=5 x^{2}-9 y^{2}$ and $f_{3}=x^{4}+6 x^{3}+12 x^{2}+6 x-5 y^{2}$ which are not equivalent and also do not have any common subexpression over $\mathbb{Z}$. After computing $f_{1}=(x+5 y) \bmod 4=x+y$, $f_{2}=\left(5 x^{2}-9 y^{2}\right) \bmod 4=x^{2}-y^{2}=(x+y)(x-y)$ and $f_{3}=x^{4}+6 x^{3}+12 x^{2}+6 x-5 y^{2} \bmod 4=x^{2}-y^{2}=(x+y)(x-y)$ using Modular-Horner Expansion Diagrams (HED) [1], it is obvious that $\left(f_{2} \bmod 4=f_{3} \bmod 4\right)$ and a common term, i.e. $x+y$, exists over $\mathbb{Z}_{4}$. This common term can be shared between the implementations of $f_{1}, f_{2}$ and $f_{3}$.

\section{A. Our Contributions}

Let $f_{1}(\vec{x}), \ldots, f_{s}(\vec{x})$ be $s$ given polynomial functions over $\mathbb{Z}_{2^{n}}$ where $\vec{x}=\left(x_{1}, x_{2}, \ldots, x_{d}\right)$ is a vector of $d$ input variables and

This reseach work was supported in part by the German Academic Exchange Service (DAAD) under grant number D/09/02091. $n$ is the word-length of each variable. This paper concentrates on finding factorizations of these functions so that a maximal number of monomials is shared between the $s$ given polynomial functions over $\mathbb{Z}_{2^{n}}$ in order to optimize the area as much as possible. We propose an algorithm that is able to symbolically factorize a set of functions, $f_{i}(i=1, \ldots, s)$, by factorizing $f_{i}$ into three sub-polynomials $p_{1, i}$, $p_{2, i}$ and $p_{3, i}$ so that $f_{i}=p_{1, i} \times p_{2, i}+p_{3, i}$, where coefficients are later matched to new, less expensive values. This optimization is done with respect to a cost function, that estimates area in terms of multipliers or adders. While the structure of the decomposition is similar to previous heuristics, in conclusion, our main contributions in this paper are as follows:

- Using symbolic factorization

- Minimization using formal methods

- Optimal results with respect to the cost function

\section{B. Structure}

This paper is structured as follows. The next two sections give an overview of related work in the area of polynomial minimization and introduce some definitions and notations used in this paper, respectively. Afterwards in Section IV and Section V the single- and multi-polynomial optimization algorithms are described. Experimental results are presented in Section VI followed by a summary of this work in Section VII.

\section{RELATED WORK}

Although a lot of work has been done to perform optimizations in the context of code generation techniques [2], the presented algorithms do not efficiently reduce the number of operations in a set of arithmetic expressions. Some work was done to optimize code with arithmetic expressions by factorization of the expressions [3]. This work developed a canonical form for representing the arithmetic expressions and an algorithm for finding common subexpressions. The drawback of this algorithm is that it takes advantage of only the associative and commutative properties of arithmetic operators and therefore can only optimize expressions consisting of a single type of associative and/or commutative operator at a time. As a result, it cannot generate complex common sub-expressions consisting of additions and multiplications. Moreover, this approach does not provide a modular factorization over $\mathbb{Z}_{2^{n}}$.

The Horner form is a well-known representation of polynomial expressions and is the most straightforward way of evaluating polynomial approximations of trigonometric functions in many libraries. This method transforms the expression into a sequence of nested additions and multiplications, which are suitable for sequential machine evaluation using Multiply-Accumulator (MAC) instructions. In spite of its advantages in sequential implementations, it does not provide an efficient optimization for combinational multivariate polynomials. 
As an example, using the Horner form, the polynomial $2 x^{2} z+6 x y z$ is factorized as $x(2 x z+6 y z)$.

Symbolic computer algebra based manipulation [3], [4], [5] and factorization with Common Sub-expression Elimination (CSE) [6] are much better approaches in optimizing polynomials compared to the Horner form. For instance, the function $2 x^{2} z+6 x y z$ is reduced to $x z(2 x+6 y)$, using the CSE method. We can enhance this approach with a coefficient factorization to obtain $2 x z(x+3 y)$. Moreover, CSE can be combined with the Modular-HED to provide more efficient polynomials over $\mathbb{Z}_{2^{n}}$ [1]. Despite these advantages, the CSE method is unable to efficiently factorize some sort of polynomials. For example $x^{2}+6 x y+9 y^{2}$ is factorized as $x(x+6 y)+9 y^{2}$ if we employ the kernel/co-kernel extraction with CSE [6], [7]. A better factorization for this polynomial is $(x+3 y)^{2}$.

The approximate factorization algorithm in [6] is an efficient approach in representing an arithmetic function $f$ as a product of sub-functions: $f=f_{1} \times f_{2} \times \ldots \times f_{N}$, where $f_{i}$ is a multivariate polynomial. However, this approach is able to factorize square-free polynomials and cannot deal with a sub-function $f_{i}$ with a degree higher than one. An example is the polynomial $(x+3 y)^{2}$, which includes a sub-function with the degree of two. Regarding such polynomials, the method in [8] has to be enhanced with techniques to initially reduce the degree of all the sub-functions to one. Another important drawback of this method is that it cannot handle those polynomials which are not reducible to $f=f_{1} \times f_{2} \times \ldots \times f_{N}$. As an example the function $x^{2}+6 x y+9 y^{2}+2 z$ cannot be reduced to the function $(x+3 y)^{2}+2 z$ using [8], because we need to leave out the monomial $z$. There have been some efforts in the area of polynomial optimization. However, they are limited in their capability to employ sophisticated manipulations to reduce the cost of the implementation.

Algebraic techniques in [9], [10] employed various optimization techniques to manipulate the polynomials and extract common subexpressions. The technique in [10] first extracts coefficient multiplications. Then using the kernel/co-kernel extraction techniques from [7] and [11], common cubes are extracted. Using these extraction techniques, a large number of linear blocks is exposed. Finally, algebraic division is performed to determine whether the obtained linear blocks are good divisors for optimizing the hardware implementation. Despite these advantages, this technique is only applicable to those polynomials in which linear blocks exist explicitly. For example, this technique is not able to decompose $x^{3}+y^{3}$ because $(x+y)$ does not exist in the given polynomial as a linear term.

Another algebraic method has been proposed in [12] and then improved in [13]. The main idea is similar to algebraic division techniques used in logic synthesis. This technique tries to decompose the original polynomial poly as $p_{1} \times p_{2}+p_{3}$ while $p_{3}$ should be minimized. For doing so, all possible initial values of $p_{1}$ and $p_{2}$ must be evaluated. Then for each initialization it is necessary to check whether other monomials in poly can be represented in the form $p_{1} \times p_{2}$. Finally, the best initialization, which constitutes the lowest complexity $p_{3}$, is chosen.

The presented paper abstracts the optimization heuristics in [12], [13] to employ a symbolic partitioning algorithm and to use formal methods to find a minimal factorization with respect to the cost function. The algorithm is later extended to work on multiple polynomials.

\section{PRELIMINARIES}

This section defines the terms necessary for subsequent parts of the paper. The following definitions of monomial, term and polynomial will be used.

Definition 1: $m=\prod_{j=1}^{d} x_{j}^{P_{j}}$ is called a monomial with $d$ variables, where $x_{j}$ is an input variable, $P_{j}$ is the degree of $x_{j}$ in $m$, and $P_{j}$ is a non-negative integer value.
Definition 2: poly $=\sum_{i=1}^{M} k_{i} \times m_{i}=\sum_{i=1}^{M} t_{i}$ is called a multivariate polynomial with $\mathrm{M}$ terms $\left(t_{i}=k_{i} \times m_{i}\right)$, where $k_{i}$ and $m_{i}$ are a constant coefficient and a monomial, respectively.

To work on elements of polynomials in the above representation or in other representations, e.g. factorized, some polynomial-specific sets are defined.

Definition 3 ( $\left.T_{\text {poly }}, T_{\text {poly }}^{L}, M_{\text {poly }}, M_{\text {poly }}^{L}\right)$ : Given a polynomial in the form poly $=\sum_{i=1}^{M} t_{i}$ the set $T_{\text {poly }}=\left\{t_{1}, \ldots, t_{M}\right\}$ contains all terms in the polynomial. For a polynomial poly' in another representation the literal terms $T_{\text {poly }}^{L}$ containing terms $t_{i}$ and subpolynomials $p_{j}, p_{k}$ are defined inductively based on the syntactic structure:

$$
\begin{aligned}
T_{t_{i}}^{L} & :=\left\{t_{i}\right\} \\
T_{p_{j}+p_{k}}^{L} & :=T_{p_{j}}^{L} \cup T_{p_{k}}^{L} \\
T_{p_{j} * p_{k}}^{L} & :=T_{p_{j}}^{L} \cup T_{p_{k}}^{L}
\end{aligned}
$$

The sets $M_{\text {poly }}:=\left\{m \mid(k, m) \in T_{\text {poly }}\right\}$ and $M_{\text {poly }}^{L}:=\{m \mid(k, m) \in$ $\left.T_{\text {poly }}^{L}\right\}$ contain all monomials and literal monomials in poly, respectively.

In this work it is necessary to know the coefficient of a monomial in a polynomial.

Definition $4(\operatorname{coeff}($ poly,$m))$ : Given a polynomial poly = $\sum_{i=1}^{M} k_{i} \times m_{i}$

$$
\operatorname{coeff}(\text { poly }, m):= \begin{cases}k & (k, m) \in T_{\text {poly }} \\ 0 & \text { otherwise }\end{cases}
$$

denotes the coefficient of $m$ in poly.

This means coeff $\left(x^{3}+3 x, x\right)=3$ and $\operatorname{coeff}\left(-4 x^{4}+(a+\right.$ b) $\left.x^{2} y, x^{2} y\right)=a+b$.

Definition $5(C(m), C($ poly $))$ : The complexity of a given monomial $m_{i}=\prod_{j=1}^{d} x_{j}^{P_{i, j}}$ is denoted by $C\left(m_{i}\right)=\sum_{j=1}^{d} P_{i, j}$. The complexity of a polynomial poly is the highest complexity of a monomial in poly: $C($ poly $)=\max _{m \in M_{\text {poly }}} C(m)$.

In other words, the complexity of a monomial is the number of variable usages: $C\left(x^{2} y^{3}\right)=C(x x y y y)=5$.

Definition $6(\operatorname{sub}(m))$ : For any monomial $m=\prod_{j=1}^{d} x_{j}^{P_{j}}$ the set

$$
\operatorname{sub}(m)=\left\{m^{\prime} \mid m^{\prime}=\prod_{j=1}^{d} x_{j}^{P_{j}^{\prime}} \wedge \forall j \in\{1, \ldots, d\}: P_{j}^{\prime} \leq P_{j}\right\}
$$

contains all sub-monomials of $m_{i}$ (including $m_{i}$ ).

The set of all sub-monomials for $x^{2} y^{2} z$ is

$$
\begin{aligned}
\operatorname{sub}\left(x^{2} y^{2} z\right)= & \left\{1, x, x^{2}, x^{2} y, x^{2} y^{2}, x^{2} y^{2} z, x^{2} y z, x^{2} z, x y, x y^{2},\right. \\
& \left.x y^{2} z, x y z, x z, y, y^{2} z, y z, z\right\}
\end{aligned}
$$

Therefore $\operatorname{sub}(m)$ contains $\prod_{j=0}^{d}\left(P_{j}+1\right)$ elements for any monomial $m=\prod_{j=1}^{d} x_{j}^{P_{j}}$.

Definition 7: Given two sets $A, B$ of monomials the set $A \times B=$ $\left\{m_{a} \times m_{b} \mid m_{a} \in A \wedge m_{b} \in B\right\}$ is called monomial set multiplication.

Furthermore the notation $z=\operatorname{ite}(b, x, y)$ is used as abbreviation for the if-then-else expression:

$$
z=\operatorname{ite}(b, x, y)= \begin{cases}x & \text { if } b \\ y & \text { otherwise }\end{cases}
$$

\section{Single-Output Polynomial Optimization Algorithm}

The algorithm works on a symbolic representation of polynomials in terms of constraints. By this a constraint solver can be applied to find equivalent polynomials. A cost function guides the solver in finding area efficient solutions. Just like the polynomial, the 
cost function is represented by constraints, counting the number of multipliers or adders respectively.

This section explains the steps for the formal polynomial optimization algorithm. The basic idea is to recursively decompose a given polynomial, poly, into three sub-polynomials, $p_{1}, p_{2}$ and $p_{3}$, such that poly $=p_{1} \times p_{2}+p_{3}$. As a decomposition into $p_{1} \times p_{2}$ is not always possible, the compensation term, $p_{3}$, is subtracted from the original polynomial to make it decomposable as poly $-p_{3}=p_{1} \times p_{2}$. For this purpose, we propose an algorithm that consists of multiple steps. These steps are:

(1) Create a symbolic factorization poly $=p_{1} \times p_{2}+p_{3}$ that represents all possible factorizations.

(2) Derive formal constraints describing the symbolic factorization.

(3) Add constraints describing the cost function.

(4) Use a constraint solver to find a minimal factorization with respect to the cost function.

The following subsections describe the factorization steps.

\section{A. Symbolic Factorization}

As a first step a symbolically factorized polynomial

$$
\text { poly }_{\text {sym }}=p_{1} \times p_{2}+p_{3}
$$

is created. The polynomial polysym is symbolic as all coefficients in $p_{1}, p_{2}, p_{3}$ are free variables. A valuation of the coefficients of poly $_{\text {sym }}$ yields an equivalent, factorized form of poly.

To create all monomials in poly a naïve way is to take the set of all monomials in poly $M$,

$$
M=\bigcup_{m \in M_{\text {poly }}} \operatorname{sub}(m)
$$

as a basis for $p_{i}$. But as $p_{1}$ and $p_{2}$ in Equation (1) are multiplied, the complexity of the resulting monomials would exceed those in poly. Therefore a filter is applied to remove the most expensive monomials:

$$
\text { filter }(M)=\left\{m \mid m \in M \wedge C(m)<\max _{m^{\prime} \in M} C\left(m^{\prime}\right)\right\}
$$

This filter removes all monomials with the highest complexity, which may be only a single one in the simplest case. If multiple monomials have the highest complexity all of them are removed. Removing only the top monomials guarantees that all factorizations, e.g. Horner form, are still represented by poly $_{\text {sym }}$. Later even stricter filters are used to improve the run-time. With this filter function the sets of monomials $M_{1,2}=\operatorname{filter}(M)$ and $M_{3}=M_{1,2} \times M_{1,2}$ are built. The symbolic representation is then created using the free variables $a_{i}, b_{j}$ and $c_{k}$ for $p_{1}, p_{2}$ and $p_{3}$, respectively:

$$
\begin{aligned}
\text { poly }_{\text {sym }}= & p_{1} \times p_{2}+p_{3} \\
= & \left(\sum_{m \in M_{1,2}} a_{i} m\right) \times\left(\sum_{n \in M_{1,2}} b_{j} n\right)+\sum_{o \in M_{3}} c_{k} o \\
= & \left(a_{1} b_{1} m_{1}+a_{1} b_{2} m_{2}+\ldots+a_{2} b_{1} m_{2}+\ldots\right) \\
& +c_{1} m_{1}+c_{2} m_{2}+\ldots \\
= & \left(a_{1} b_{1}+c_{1}\right) m_{1}+\left(a_{1} b_{2}+a_{2} b_{1}+c_{2}\right) m_{2} \\
& +\ldots
\end{aligned}
$$

\section{B. Factorization Constraints}

Given Equation (4), the constraints for a valid factorization can be derived. For each monomial $m_{i}$ the coefficients in the non-factorized

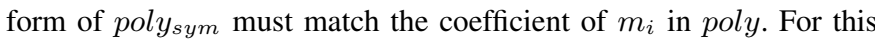
the symbolic representation in polysym is tied to the value in poly:

$$
\forall(\phi \times m) \in T_{\text {poly }_{\text {sym }}}: \phi=\operatorname{coeff}(\text { poly }, m)
$$

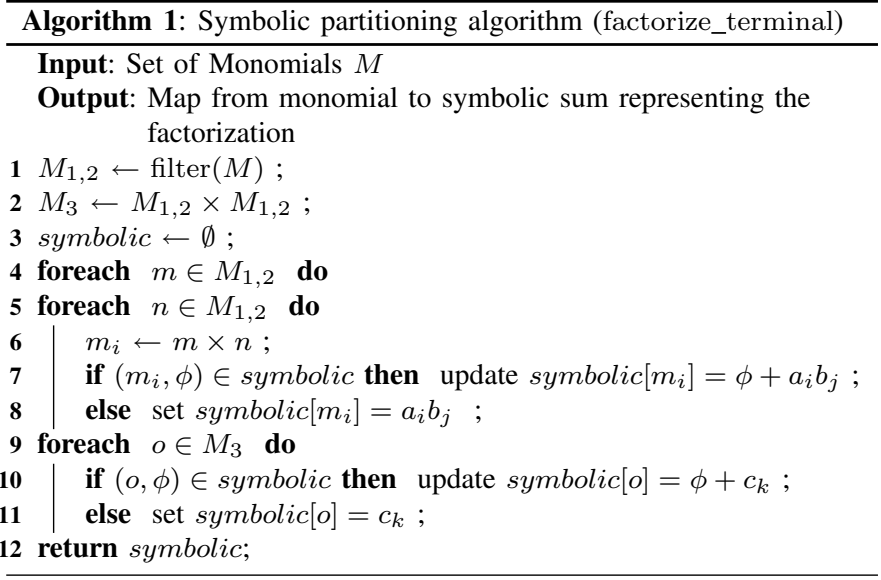

Which can be expanded to match Equation (4):

$$
\begin{array}{rlrl}
a_{1} b_{1}+c_{1} & = & \operatorname{coeff}\left(\text { poly }, m_{1}\right) \\
\wedge & a_{1} b_{2}+a_{2} b_{1}+c_{2} & = & \operatorname{coeff}\left(\text { poly }, m_{2}\right) \\
\wedge & \ldots & = & \ldots
\end{array}
$$

This means any valid assignment of $a_{i}, b_{j}$ and $c_{k}$ following the constraint in Equation (5) yields a valid factorization of poly in Equation (3).

\section{Algorithm}

Algorithm 1 creates the symbolic representation. The input to the algorithm is the set $M$ of all sub-monomials in poly as defined in Equation (2) and the return value is the map named symbolic from monomials in poly $_{\text {sym }}$ to their respective symbolic coefficients expression as in Equation (4).

In lines 1-2 the input will first be filtered and expensive monomials are removed. This is done as $p_{1}$ and $p_{2}$ are to be multiplied and the complexity as well as the number of terms of the resulting polynomial is reduced without losing any expressiveness. The remaining monomials are added to $M_{1,2}$. Afterwards $M_{3}$ is calculated as $M_{3}=M_{1,2} \times M_{1,2}$.

The double loop in line 4 and line 5 incrementally creates the product $p_{1} \times p_{2}$. In each loop one monomial is calculated (line 6) and the sum of coefficients for this monomial is updated (line 7 or line 8 , if a partial symbolic coefficient $\phi$ was already calculated or this one is new). For $p_{3}$ the loop in line 9 does the same with the monomials in $M_{3}$.

\section{Recursive Factorization}

The partitioning algorithm can also be applied recursively to find less expensive factorizations, resulting e.g. in

$$
\begin{aligned}
\text { poly }= & \left(p_{1,1} \times p_{1,2}+p_{1,3}\right) \times\left(p_{2,1} \times p_{2,2}+p_{2,3}\right) \\
& +\left(p_{3,1} \times p_{3,2}+p_{3,3}\right)
\end{aligned}
$$

where $p_{i, j}$ are either recursive themselves or terminal. Similar to the simple symbolic factorization algorithm the complexity of $p_{1, j}$ and $p_{2, j}$ is reduced in each step. The filter reduces the complexity of $p_{1}$ and $p_{2}$ by 1 in so their recursion ends in a linear number of steps. For $p_{3}$ additionally a recursion limit $d$ is used so that the algorithm is guaranteed to terminate in depth $d$. Using this approach, a symbolic factorization tree is built, as depicted in Figure 1. The nodes in the last level are called terminal and calculated according to the previous section. The nodes on higher levels are called recursive and compose three nodes from a lower level into a symbolic factorization according to Equation (6). 


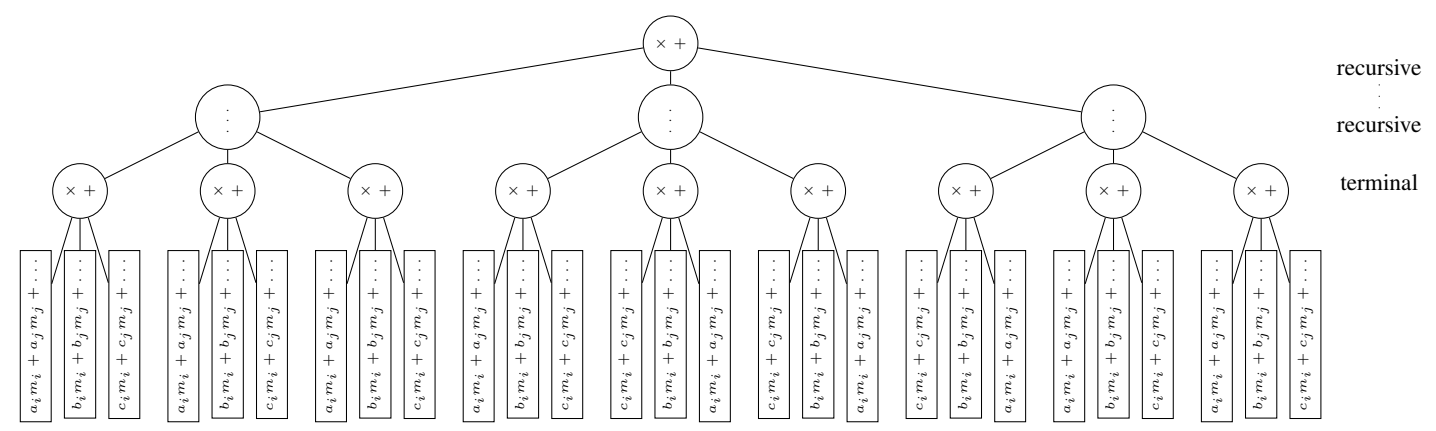

Figure 1. Recursive partitioning scheme

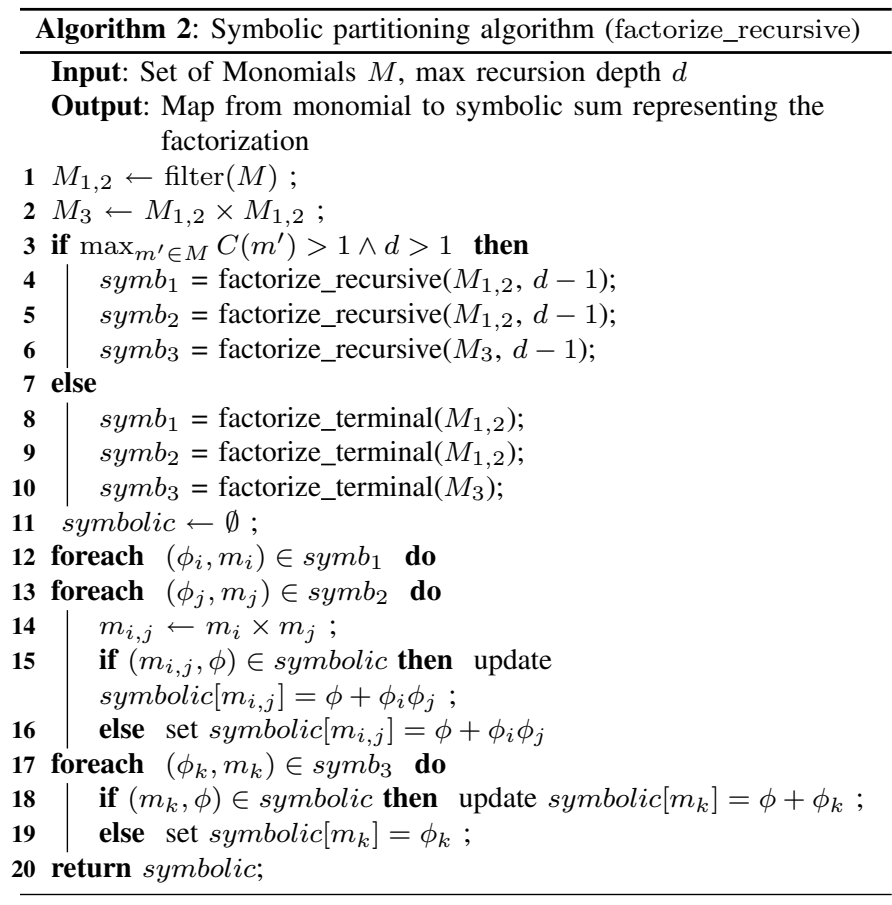

Algorithm 2 shows the details of the recursive symbolic factorization. First the monomials are filtered, like in the terminal factorization algorithm (line 1-2). In the following lines 3-6 the algorithm first checks whether to continue recursion or end with terminal factorizations. The recursion depth depends on the limit $d$ and on the complexity of the monomials. In each recursion step the limit is reduced and $M_{1,2}$ is used for both $p_{1}$ and $p_{2}$. The monomials $M_{3}$ are used for the recursion of $p_{3}$. If the recursion condition does not hold, terminal factorizations are created instead (lines 810). Afterwards, in lines 11-19 the returned symbolic factorizations are merged to get the symbolic representation in the current level. This is done in a similar way as in the terminal algorithm. Instead of variables the expressions from the recursive calls are used.

\section{E. Cost Function and Optimization}

After creating the symbolic representation an area efficient factorization has to be found, i.e. a good valuation of the variables. A constraint solver satisfying Equation (5) creates a valid assignment, i.e. a new polynomial, which is possibly more expensive than poly itself. Therefore the constraint solver has to have a notion of costs to be minimized. With respect to this cost function the most efficient factorization can be found. This section describes how to define a suitable cost function to reduce multiplications and enhance monomial reuse.
An intuitive cost function is the complexity of the used monomials, which indicates how many variables are multiplied to create the circuit. This is defined by extracting all terms from polysym and sorting the coefficient variables by monomial $m$. Let $\Gamma_{m}=\{a \mid(a, m) \in$ $\left.T_{\text {polysym }}^{L}\right\}$ contain all coefficient variables $a_{i}, b_{j}, c_{k}$ that form a term with the monomial $m$ in poly sym (see Equation (3)). Under the assumption that a monomial has to be calculated once and can be reused multiple times, the costs for the monomial are added if any of its coefficients is non-zero:

$$
\operatorname{cost}_{C}:=\sum_{m \in M_{\text {poly }}^{L} \text { sym }} \begin{cases}C(m) & \text { if } \exists a \in \Gamma_{m}: a \neq 0 \\ 0 & \text { otherwise }\end{cases}
$$

Additionally, for each symbolic partition one additional multiplier is required to create $p_{1} \times p_{2}$. For this the predicate used $\left(p_{i}\right)$ is defined to be true iff any coefficient variable in $p_{i}$ is not zero or any of the sub partitions is used. This enables the definition of cost $_{\times}$as:

$$
\begin{aligned}
\operatorname{cost}_{\times}\left(p_{i}\right):= & \operatorname{ite}\left(\operatorname{used}\left(p_{i, 1}\right) \vee \operatorname{used}\left(p_{i, 2}\right), 1,0\right) \\
+ & \operatorname{ite}\left(\text { is terminal } p_{i}, 0,\right. \\
& \left.\operatorname{cost}_{\times}\left(p_{i, 1}\right)+\operatorname{cost}_{\times}\left(p_{i, 2}\right)+\operatorname{cost}_{\times}\left(p_{i, 3}\right)\right)
\end{aligned}
$$

The overall cost of multiplications is given by

$$
\operatorname{cost}_{\text {mult }}=\operatorname{cost}_{C}+\operatorname{cost}_{\times}
$$

Example 1: Given polynomial $p=x^{2}-y^{2}$ containing the monomials and sub-monomials $x^{2}, x, y^{2}, y, 1$. The first partitioning step for $p=p_{1} \times p_{2}+p_{3}$ results in $M_{1,2}=\{x, y, 1\}$ and $M_{3}=\left\{x^{2}, x y, y^{2}, x, y, 1\right\}$. The symbolic partitioning therefore results in:

$$
\begin{aligned}
p= & \left(a_{1} x+a_{2} y+a_{3}\right) \times\left(b_{1} x+b_{2} y+b_{3}\right) \\
& +\left(c_{1} x^{2}+c_{2} x y+c_{3} y^{2}+c_{4} x+c_{5} y+c_{6}\right)
\end{aligned}
$$

From this form the constraints for factorization are derived. For each monomial in $p$ the correct sum is constrained:

$$
\begin{aligned}
a_{1} b_{1}+c_{1} & = & 1 & \left(\text { for } x^{2}\right) \\
a_{2} b_{2}+c_{3} & = & -1 & \left(\text { for } y^{2}\right) \\
a_{1} b_{2}+a_{2} b_{1}+c_{2} & = & 0 & (\text { for } x y)
\end{aligned}
$$

The corresponding cost functions are

$$
\begin{aligned}
\operatorname{cost}_{C} & :=\operatorname{ite}\left(c_{1} \neq 0,2,0\right) \\
& +\operatorname{ite}\left(c_{2} \neq 0,2,0\right) \\
& +\operatorname{ite}\left(c_{3} \neq 0,2,0\right) \\
& +\operatorname{ite}\left(a_{1} \neq 0 \vee b_{1} \neq 0 \vee c_{4} \neq 0,1,0\right) \\
& +\operatorname{ite}\left(a_{2} \neq 0 \vee b_{2} \neq 0 \vee c_{5} \neq 0,1,0\right) \\
\text { cost }_{\times} & :=\operatorname{ite}\left(\bigvee_{i=1}^{3} a_{i} \neq 0 \vee \bigvee_{j=1}^{3} b_{i} \neq 0,1,0\right)
\end{aligned}
$$


The given polynomial $p=x^{2}-y^{2}$ has costs of $\operatorname{cost}_{\text {mult }}(p)=$ $C\left(x^{2}\right)+C\left(y^{2}\right)=4$. Using a constraint-solver and the constraint cost $_{\text {mult }}\left(\right.$ poly $\left._{\text {sym }}\right) \leq 4$, the solution poly sym $_{\text {s. }}=(x+y) \times(x-y)$ is found with minimal costs of 3 .

After minimizing the number multipliers, an additional step is performed to minimize the number of adders. Similar to cost $_{m u l t}$, the number of additions in terminal partitions plus additions introduced through (recursive) partitioning is defined to be the cost of addition cost $_{\text {add }}$. Adder structures are used in terminal parts $p_{1}, p_{2}, p_{3}$ to sum up all terms. Therefore the number of terms $n$ is counted and $n-1$ adders are needed for $p_{i}$ if at least two terms have non-zero coefficients:

$$
\operatorname{adders}(p)=\max \left(1, \sum_{(a, m) \in T_{p}} \operatorname{ite}(a \neq 0,1,0)\right)-1
$$

Additionally for each symbolic partition one adder is used with $\left(p_{1} \times p_{2}\right)+p_{3}$. The number of adders is therefore:

$$
\begin{aligned}
\operatorname{cost}_{+}\left(p_{i}\right):= & \operatorname{ite}\left(\left(\operatorname{used}\left(p_{i, 1}\right) \vee \operatorname{used}\left(p_{i, 2}\right)\right) \wedge \operatorname{used}\left(p_{i, 3}\right), 1,0\right) \\
+\quad & \operatorname{ite}\left(\text { is terminal } p_{i}\right. \\
& , \operatorname{adders}\left(p_{i, 1}\right)+\operatorname{adders}\left(p_{i, 2}\right)+\operatorname{adders}\left(p_{i, 3}\right) \\
& \left., \operatorname{cost}_{+}\left(p_{i, 1}\right)+\operatorname{cost}_{+}\left(p_{i, 2}\right)+\operatorname{cost}_{+}\left(p_{i, 3}\right)\right)
\end{aligned}
$$

We apply a two-step algorithm. The first step is to minimize the costs of multiplication. Afterwards cost mult $_{\text {is }}$ is constrained to the optimal value. In the second step cost $_{+}$, i.e. the number of adders is minimized.

Other types of cost functions are also possible, e.g. a single-step optimization that considers adder and multiplier costs in a single cost function, e.g. by using the area required for each type. Furthermore modelling other targets as delay or common sub-polynomials is possible. On the other hand more complex cost function will typically increase the run-time.

As a constraint solver an (incremental) solver for Satisfiability Modulo Theories (SMT) for bit-vector theory [14] is used. Such a solver can naturally represent constraints over $\mathbb{Z}_{2^{n}}$ including modulo arithmetics and can efficiently find valid assignments or prove that no such assignment exists.

The factorization constraints are translated to SMT bit-vector logic with the symbolic coefficients as bit-vector variables. Furthermore the cost function is expressed in terms of these variables. The minimization algorithm uses multiple calls to the solver until the minimum is found. For this the algorithm assumes a cost value as upper bound, e.g. costs of input polynomial poly, and queries the solver whether a solution with costs less than the upper bound exists. In practice binary search using a lower and an upper bound was most efficient for finding the minimal cost value. The bounds are updated incrementally. If the constraint cost $<c$ is satisfiable, the upper bound is updated, otherwise the lower bound is updated. The value $c$ in the next iteration is calculated as $c=\frac{u p p e r+\text { lower }}{2}$. When upper $=$ lower holds, the minimum is found.

This algorithm always finds the minimal value of the cost function. Finding an optimal solution is expected to result in high run-times, therefore two restrictions were applied to reduce the size of the constraint solver instance:

- The factorization of $p_{3}$ is terminal for all cases.

- A more restrictive filter was applied that eliminates the top half of the monomials in each step.

This significantly decreases the run-time of the SMT solver, but excludes some possible factorizations.

\section{Multi-Output Polynomial Optimization Algorithm}

The single-output polynomial optimization algorithm can be extended to minimize the multipliers of several polynomials at the same time. This is based on the same assumption, that a monomial calculated once can be shared in multiple polynomials. This means that a monomial $m$ used in poly $y^{(1)}$ and poly ${ }^{(2)}$ has to be synthesized only once.

The basic task is to find a good representation for a set of $n$ polynomials polys $=\left\{\right.$ poly ${ }^{(1)}, \ldots$, poly $\left.^{(n)}\right\}$. The single-output polynomial optimization algorithm is extended to minimize such a set. The symbolic representation does not change, it is applied on each polynomial separately. For each polynomial poly ${ }^{(i)}$ the symbolic representation poly $y_{s y m}^{(i)}$ is created according to Equation (3). Afterwards the respective factorization constraints as in Equation (5) are derived.

Only the cost function has to be adapted. The complexity costs are calculated over the monomials from all polynomials:

$$
\begin{aligned}
\Gamma_{m}^{\prime} & =\left\{a \mid(a, m) \in \bigcup_{i=1}^{n} T_{\text {polysym }}^{L}\right\} \\
\operatorname{cost}_{C}^{\prime} & =\sum_{\left.m \in \bigcup_{i=1}^{n} M_{\substack{M_{\text {oly }}^{L} \\
\text { poym }}}^{(i)}\right\}} \begin{cases}C(m) & \text { if } \exists a \in \Gamma_{m}^{\prime}: a \neq 0 \\
0 & \text { otherwise }\end{cases}
\end{aligned}
$$

Hence, the overall cost of multiplications are now:

$$
\operatorname{cost}_{\text {mult }}^{\prime}=\operatorname{cost}_{C}^{\prime}+\sum_{p \in \text { polys }} \operatorname{cost}_{\times}(p)
$$

To find an optimal assignment the same algorithm is applied as for the optimization of single-output polynomials. Note also that the multi-polynomial algorithm collapses to the single-polynomial algorithm when applied to only one polynomial. Considering the single polynomial is therefore only a special case of the general algorithm.

\section{EXPERIMENTAL RESULTS}

The experiments were run on an Intel Core 2 Duo CPU at $3.33 \mathrm{GHz}$ with $3 \mathrm{~GB}$ of main memory running a Linux operating system. As SMT-Solver Boolector 1.1 was used [15]. In order to demonstrate the advantage of our proposed algorithms over the stateof-the-art techniques, we use several polynomials extracted from real embedded systems such as digital signal processing, computer graphics, automotive and communication applications. In the first experiment, we have employed phase-shift keying (PSK) that is used in digital communication from [4], digital image rejection unit (DIRU), Degree-5 Filter (PFLT), multivariate cosine wavelet polynomial (MVCS), an anti-aliasing function (ANTI) [4], a Savitzky Golay filter (SG2) and Quadratic polynomial (QUAD) benchmarks and applied our method to the benchmarks listed in Table I as singleoutput polynomials. These benchmarks have been used in previous work on polynomial datapath optimization such as [13], [10]. We have synthesized the polynomials using a traditional logic synthesis tool in $0.25 \mu \mathrm{m}$ CMOS technology.

The important parameters of the circuits including the area, counted by number of logic gates (Area) as well as the critical path delay in nanoseconds (Delay) are shown in Table I. Column M/V/D/n provides the number of monomials/the number of variables/the highest degree/the bit-vector size of each variable. In each experiment multiplier and adder minimization as described previously was applied.

We compare the results of our approach to the one of [13] that has been shown to perform about $20 \%$ to $40 \%$ better than state-ofthe-art synthesis approaches. Even with the run-time optimizations described in Section IV, our algorithm takes considerably more time 
Table I

COMPARISON OF THE FORMAL OPTIMIZATION TECHNIQUE WITH THE APPROACH IN [13] (Single-OUTPUT POLYNOMIALS)

\begin{tabular}{|c|c|c|c|c|c|c|c|}
\hline \multirow[t]{2}{*}{ Benchmark } & \multirow[t]{2}{*}{$\mathrm{M} / \mathrm{V} / \mathrm{D} / \mathrm{n}$} & \multicolumn{3}{|c|}{ Technique in [13] } & \multicolumn{3}{|c|}{ SMT-based } \\
\hline & & $\begin{array}{l}\text { Time } \\
(\mathrm{s})\end{array}$ & $\begin{array}{l}\text { Delay } \\
\text { (ns) }\end{array}$ & Area & $\begin{array}{l}\text { Time } \\
\text { (s) }\end{array}$ & $\begin{array}{l}\text { Delay } \\
\text { (ns) }\end{array}$ & Area \\
\hline ANTI & $7 / 1 / 6 / 16$ & $<0.01$ & 38.78 & 100574 & 15.4 & 34.83 & 88761 \\
\hline DIRU & $8 / 2 / 4 / 16$ & 3.10 & 23.17 & 55631 & 57.9 & 20.57 & 48501 \\
\hline MVCS & $9 / 2 / 3 / 16$ & 2.60 & 27.49 & 111801 & 12,7 & 23.89 & 95147 \\
\hline PFLT & $6 / 1 / 5 / 16$ & $<0.01$ & 39.38 & 76069 & 15.5 & 34.87 & 65819 \\
\hline PSK & $9 / 2 / 4 / 16$ & 4.00 & 29.24 & 141923 & 24.2 & 35.24 & 143277 \\
\hline QUAD & $5 / 2 / 2 / 16$ & $<0.01$ & 21.64 & 48289 & 3.8 & 18.14 & 45090 \\
\hline SG2 & $9 / 2 / 3 / 16$ & $<0.01$ & 29.21 & 149496 & 6.4 & 25.58 & 127578 \\
\hline \multicolumn{2}{|l|}{ aver } & & & & & $7.72 \%$ & $10.47 \%$ \\
\hline
\end{tabular}

than [13]. This is not surprising, since we are comparing heuristic and exact algorithms. In all cases but one a significantly lower number of gates is achieved compared to [13]. As a side-effect the delay is also reduced on the same instances. The slightly poorer performance on PSK instance is due to the fact that the heuristic [13] is able to extract two sub-polynomials in the form of $p_{1}^{\prime} \times p_{2}^{\prime}+p_{3}^{\prime} \times p_{4}^{\prime}+p_{5}^{\prime}$. This form is covered by recursive factorization of $p_{3}$ in $p_{1} \times p_{2}+p_{3}$ as $p_{1}^{\prime} \times p_{2}^{\prime}+\left(p_{3}^{\prime} \times p_{4}^{\prime}+p_{5}^{\prime}\right)$. But as already described, recursive factorization of $p_{3}$ was disabled because it drastically impacts the run-time of the algorithm. On average a reduction of $10.47 \%$ in area and by $7.72 \%$ in delay is achieved.

In another experiment, we have employed different combinations of PSK, QUAD, MVCS, PFLT, DIRU, ANTI and SG2 benchmarks as multi-output polynomials. In addition to the results from [13] we also included results from [10]. Table II summarizes the results for 22 configurations. Our results show that for a given set of polynomial expressions, our approach determines better factorizations for all combinations but one with respect to the area. In instance 14, the heuristics outperform our algorithm. The technique in [13] is able to extract a common expression $(x+y)$ from multiple polynomials. Our algorithm cannot find this extraction as it does not result in an improvement of the cost function, i.e. the cost of multiplication does not decrease. On the other hand we are able to reduce the area on instance 20 by $30.93 \%$ and its delay by $15.17 \%$ compared to [13]. Furthermore on instance 5 we achieve a reduction of $46.14 \%$ in delay and $16.87 \%$ in area compared to [10]. Overall we obtained average savings of $12.15 \%$ in the area and $3.05 \%$ in the delay over [13]. Compared to [10] we achieved average savings of $21.17 \%$ in the delay and $5.72 \%$ in the area.

Table II

COMPARISON OF THE FORMAL OPTIMIZATION TECHNIQUE WITH THE APPROACHES IN [10] AND [13] (MULTI-OUTPUT POLYNOMIALS)

\section{CONCLUSIONS}

This paper presented a methodology to describe polynomial factorization and minimization as a constraint solving problem. This methodology was extended to recursive factorization and multiple polynomials. The approach outperforms state-of-the-art optimization techniques. On average the algorithm improves area and delay for the benchmarks considered.

Future extensions of this approach are the realization of different cost functions such as delay as well as further run-time optimizations.

\section{REFERENCES}

[1] B. Alizadeh and M. Fujita, "Modular-HED: A canonical decision diagram for modular equivalence verification of polynomial functions," in Proc. of 5th International Workshop on Constraints in Formal Verification (CFV), 2008, pp. 22-40.

[2] A. V. Aho, S. C. Johnson, and J. D. Ullman, "Code generation for expressions with common subexpressions," Journal of the ACM, vol. 24 no. 1, pp. 146-160, 1977

[3] M. A. Breuer, "Generation of optimal code for expressions via factorization," Comm. of the ACM, vol. 12, no. 6, pp. 333-340, 1969.

[4] A. Peymandoust and G. D. Micheli, "Application of symbolic computer algebra in high-level data-flow synthesis," IEEE Trans. on CAD, vol. 22, no. 9, pp. 1154-1165, 2003.

[5] S. Gopalakrishnan and P. Kalla, "Optimization of polynomial datapaths using finite ring algebra," ACM Trans. on Design Automation of Electronic Systems, vol. 12, no. 4, p. 49, 2007.

[6] A. Hosangadi, F. Fallah, and R. Kastner, "Factoring and eliminating common subexpressions in polynomial expressions," in Int'l Conf. on $C A D, 2004$, pp. 169-174.

[7] B. DeRenzi and W. Gong, "JuanCSE," 2005, http://express.ece.ucsb. edu/suif/cse.html, last updated 2005. [Online]. Available: \url\{http: //express.ece.ucsb.edu/suif/cse.html\}

[8] E. Kaltofen, J. P. May, Z. Yang, and L. Zhi, "Approximate factorization of multivariate polynomials using singular value decomposition," $J$. Symb. Comput., vol. 43, no. 5, pp. 359-376, 2008.

[9] S. Gopalakrishnan, P. Kalla, M. B. Meredith, and F. Enescu, "Finding linear building-blocks for RTL synthesis of polynomial datapaths with fixed-size bit-vectors," in Int'l Conf. on CAD, 2007, pp. 143-148.

[10] S. Gopalakrishnan and P. Kalla, "Algebraic techniques to enhance common sub-expression elimination for polynomial system synthesis," in Design, Automation and Test in Europe, 2009, pp. 1452-1457.

[11] A. Hosangadi, F. Fallah, and R. Kastner, "Optimizing polynomial expressions by algebraic factorization and common subexpression elimination," IEEE Trans. on CAD, vol. 25, no. 10, pp. 2012-2022, 2006.

[12] O. Sarbishei, B. Alizadeh, and M. Fujita, "Polynomial datapath optimization using partitioning and compensation heuristics," in Design Automation Conf. ACM, 2009, pp. 931-936.

[13] B. Alizadeh and M. Fujita, "Improved heuristics for finite word-length polynomial datapath optimization," in Int'l Conf. on CAD. ACM, 2009, pp. 739-744.

[14] S. Ranise and C. Tinelli, "The Satisfiability Modulo Theories Library (SMT-LIB)," http://www.smtlib.org, 2006.

[15] R. Brummayer and A. Biere, "Boolector: An efficient SMT solver for bit-vectors and arrays," in Tools and Algorithms for the Construction and Analysis of Systems, 2009, pp. 174-177.

\begin{tabular}{|c|c|c|c|c|c|c|c|c|c|c|}
\hline \multirow[t]{2}{*}{ Number } & \multirow[t]{2}{*}{ Benchmark } & \multirow[t]{2}{*}{$\mathrm{M} / \mathrm{V} / \mathrm{D} / \mathrm{n}$} & \multicolumn{2}{|c|}{ Technique in [10] } & \multicolumn{3}{|c|}{ Technique in [13] } & \multicolumn{3}{|c|}{ SMT-based } \\
\hline & & & Delay (ns) & Area & Time (s) & Delay (ns) & Area & Time (s) & Delay (ns) & Area \\
\hline 1 & DIRU, PFLT & $14 / 2 / 5 / 16$ & 38,23 & 117357 & 3.10 & 26.87 & 110362 & 35.60 & 21.55 & 106220 \\
\hline 2 & PSK, PFLT & $15 / 2 / 5 / 16$ & 31,75 & 172597 & 4.00 & 31.03 & 193366 & 18.10 & 31.99 & 184223 \\
\hline 3 & QUAD, PFLT & $11 / 2 / 5 / 16$ & 38,29 & 158975 & 0.01 & 30.88 & 123192 & 18.20 & 39.07 & 112102 \\
\hline 4 & ANTI, PFLT & $13 / 1 / 6 / 16$ & 54,30 & 143097 & 0.02 & 39.92 & 154170 & 35.80 & 40.30 & 131284 \\
\hline 5 & MVCS, PFLT & $15 / 2 / 5 / 16$ & 37,91 & 159732 & 2.60 & 27.89 & 156335 & 46.10 & 20.42 & 132791 \\
\hline 6 & DIRU, MVCS, PFLT & $23 / 2 / 5 / 16$ & 37,91 & 192217 & 5.70 & 31.90 & 208479 & 4575.70 & 34.23 & 196199 \\
\hline 7 & DIRU, PSK, PFLT & $23 / 2 / 5 / 16$ & 31,75 & 191522 & 7.10 & 29.02 & 202515 & 311.20 & 27.57 & 154228 \\
\hline 8 & DIRU, QUAD, PFLT & $19 / 2 / 5 / 16$ & 38,34 & 190864 & 3.10 & 29.88 & 176156 & 120.10 & 26.05 & 152455 \\
\hline 9 & MVCS, PSK, PFLT & $24 / 2 / 5 / 16$ & 31,75 & 215592 & 6.60 & 31.05 & 279886 & 30.70 & 32.04 & 240726 \\
\hline 10 & MVCS, QUAD, PFLT & $20 / 2 / 5 / 16$ & 38,33 & 229893 & 2.60 & 31.12 & 201089 & 68.40 & 31.76 & 188927 \\
\hline 11 & PSK, QUAD, PFLT & $20 / 2 / 5 / 16$ & 43,18 & 257574 & 4.00 & 31.04 & 200728 & 23.90 & 29.09 & 190637 \\
\hline 12 & DIRU, ANTI, PFLT & $21 / 2 / 6 / 16$ & 54,38 & 178268 & 3.10 & 39.93 & 209439 & 208.70 & 40.06 & 154724 \\
\hline 13 & DIRU, MVCS, PSK, PFLT & $32 / 2 / 5 / 16$ & 31,75 & 234004 & 9.70 & 32.06 & 286603 & 247.80 & 31.40 & 254738 \\
\hline 14 & DIRU, MVCS, QUAD, PFLT & $28 / 2 / 5 / 16$ & 38,33 & 291980 & 5.70 & 32.90 & 283855 & 479.30 & 37.82 & 445008 \\
\hline 15 & DIRU, PSK, QUAD, PFLT & $28 / 2 / 5 / 16$ & 43,07 & 249654 & 7.10 & 30.02 & 248303 & 12512.20 & 31.74 & 221264 \\
\hline 16 & MVCS, PSK, QUAD, PFLT & $29 / 2 / 5 / 16$ & 43,08 & 274683 & 6.60 & 30.96 & 304841 & 67.50 & 31.32 & 271477 \\
\hline 17 & DIRU, MVCS, ANTI, PFLT & $30 / 2 / 6 / 16$ & 54,32 & 251844 & 5.70 & 39.93 & 317693 & 73.90 & 34.37 & 248499 \\
\hline 18 & DIRU, MVCS, PSK, ANTI, PFLT & $39 / 2 / 6 / 16$ & 54,48 & 408720 & 9.70 & 39.86 & 410926 & 4272.20 & 33.19 & 324402 \\
\hline 19 & DIRU, MVCS, PSK, SG2, PFLT & $41 / 2 / 5 / 16$ & 41,71 & 339997 & 9.70 & 39.56 & 458484 & 6167.30 & 35.56 & 326957 \\
\hline 20 & DIRU, MVCS, PSK, QUAD, SG2, PFLT & $46 / 2 / 5 / 16$ & 41,71 & 389744 & 9.70 & 39.56 & 498052 & 6289.30 & 33.56 & 343990 \\
\hline 21 & DIRU, MVCS, PSK, SG2, ANTI, PFLT & $48 / 2 / 6 / 16$ & 54,59 & 394574 & 9.70 & 39.86 & 529409 & 759.10 & 36.94 & 428977 \\
\hline 22 & DIRU, MVCS, PSK, QUAD, SG2, ANTI, PFLT & $53 / 2 / 6 / 16$ & 54,59 & 434178 & 9.70 & 39.86 & 568499 & 1421.60 & 41.27 & 409942 \\
\hline \multicolumn{3}{|c|}{ average savings: } & $21.17 \%$ & $5.72 \%$ & & $3.05 \%$ & $12.15 \%$ & & & \\
\hline
\end{tabular}

